\title{
Observership in Shanghai, China-meeting emerging leading pioneers of robotic thoracic surgery and ERAS
}

\author{
Beatrice Chia-Hui Shih \\ Department of Cardiovascular and Thoracic Surgery, Seoul National University Hospital, Seoul National University College of Medicine, Seoul, \\ South Korea \\ Correspondence to: Beatrice Chia-Hui Shih. Department of Cardiovascular and Thoracic Surgery, Seoul National University Hospital, Seoul National \\ University College of Medicine, Seoul, South Korea. Email: beatriceshih@gmail.com.
}

\begin{abstract}
China is irrefutably a growing giant in thoracic surgery with unparalleled number of surgical cases and increasing academic researches given the rapidly accumulated data. The ESTS and the AME Publishing Group enable exciting opportunity to exchange ideas, discuss new clinical techniques, and foster rich experience in surgical education through valuable international observership award. This year's visiting scholarship allows collaboration between South Korea and China, two very close but disparate countries. This is a perspective paper discussing the current thoracic surgery training in South Korea and it attempts to outline objectives in visiting scholarships from a third-year resident's point of view.
\end{abstract}

Keywords: Thoracic surgery training; Asia; China; Korea; Observership; visiting scholarship

Submitted Oct 06, 2018. Accepted for publication Oct 13, 2018.

doi: $10.21037 /$ jtd.2018.11.79

View this article at: http://dx.doi.org/10.21037/jtd.2018.11.79

\section{Introduction}

During the postgraduate course Master's Cup competition at the $26^{\text {th }}$ European Conference on General Thoracic Surgery, one month observership in Shanghai Ruijin Hospital was awarded for best performance to a trainee member. With sponsorship from the AME Publishing Company, this visiting scholarship allows valuable academic and cultural exchange between two closely similar yet different countries in Asia-South Korea and China. This is a personal perspective written in advance to actual visit, outlining what the author (Beatrice C. Shih) expects to achieve and see from this academic collaboration. Unfortunately, due to recent changes in Korean residency training policy, the duration of observership is shortened to half of what was originally intended.

\section{Thoracic surgery in South Korea and what China offers}

After obtaining medical license since graduation from medical school, newly established doctors serve one year of internship in a selected hospital before choosing to pursue a career in a specific field. In South Korea, current cardiovascular and thoracic surgery training lasts 4 years; first two years of residency usually focus on preoperative and postoperative patient management, learning general surgical techniques, and research techniques. Latter two years of residency focus on critical care management in intensive care units (ICU) and surgical skills in operative theatre. In Seoul National University Hospital (SNUH) Thoracic Surgery department, thoracic surgical oncology is the main theme of the division. About 800 cases of lung cancer and 100 cases of esophageal cancer surgery are performed every year, and by 2016, cumulative number of lung cancer surgery approached 5,000 cases. Visiting scholarship is one of the resident training paradigm in Korea. The SNUH cardiothoracic training integrates a 2 -week visiting fellowship to any leading institution in the world to $3^{\text {rd }}$ year residents. However, as the number of residents has always been on demand and with the newly implemented 80-hour Resident Workweek policy by the South Korean government, the need for exposure to efficient and greater number of academic and surgical experience in a shorter 
period of time has become imminent. On the other hand, China provides a great opportunity for residents training in thoracic surgery. The advent of ultra-high volume thoracic surgical centers in Shanghai allows trainees to absorb incredible amount of surgical experience in a given short period of time $(1,2)$. In other words, this is what China can offer: vast number of operation cases and unparalleled number of collective data for academic research.

\section{Observership in Shanghai}

The author will spend two weeks in Shanghai Ruijin Hospital in China with three big objectives in mind. First, keeping in mind that the Shanghai Ruijin Hospital performed more than 600 robot assisted surgeries as of March 2018 (3), she seeks to learn host institution's unique aspects of minimally invasive surgery in many challenging and interesting cases. Even with a short visit, a trainee would be able to gain an incredible amount of live experience, that allows a balanced perspective on thoracic surgery of various diseases. Successful and efficient implementation of early recovery after surgery (ERAS) is also of another author's interest. With huge number of patient inflow and operations performing in single center, the role of clinically efficient routine is critical. The host institution has seamlessly connected the ERAS program with Rehabilitation Department and how this multidisciplinary pre and postoperative effort can be done in such huge volume center is of question. Apart from this clinical aspect, how the large number of patient flow is efficiently managed in outpatient clinics is also of administrative interest. The outpatient clinic is no longer a part of training paradigm in the author's hospital after the advent of 80-hour Workweek Policy-therefore, participating in outpatient clinic will enable interesting discussion on screening, planning, and coordinating the operation in demographically diverse patients in China $(1,2)$. Lastly, the author would like to find out means of developing multi-institutional research collaboration between South Korea and China, given the advantages in geographic proximity.

During the observership, the author will be attending outpatient clinics and various surgical cases of Dr. Hecheng $\mathrm{Li}$, hoping to exchange interactive dialogue and discussion. In addition, she will be participating in grand rounds and journal meetings, where she will be presenting on the South Korean residency training in cardiovascular and thoracic surgery.

The author hopes that this observership opportunity would not be limited to a single trainee member in the future, and serve as an academic initiative towards collaborative research between the two centers.

\section{Acknowledgements}

The author would like to thank Professor Sanghoon Jheon, Kwanmienne Kim, Sukki Cho, Jaehyun Jheon from South Korea and Professor Hui Li from China, for providing the opportunity to participate in the Postgraduate Master's Cup at the $26^{\text {th }}$ ESTS, Ljubliana Slovenia. The author also gives thanks to the AME Publishing Group and ESTS for making this international observership possible albeit political difficulties.

\section{Footnote}

Conflicts of Interest: The author has no conflicts of interests to declare.

\section{References}

1. Sihoe AD. Opportunities and challenge for thoracic surgery collaborations in China: a commentary. J Thorac Dis 2016;8:S414-26.

2. Sihoe AD. The Advent of Ultra-high volume thoracic surgical centers in Shanghai. World J Surg 2017;41:2758-68.

3. Xu E. Hecheng Li: sticks with the spirit of craftsmanship. J Thorac Dis 2018;10:S1290-6.
Cite this article as: Shih BC. Observership in Shanghai, China-meeting emerging leading pioneers of robotic thoracic surgery and ERAS. J Thorac Dis 2018;10(12):E806-E807. doi: 10.21037/jtd.2018.11.79 